\title{
Triggering receptor expressed on myeloid cells 1 and 2 in patients with chronic maxillary sinusitis
}

\author{
Tedla $\mathrm{M}^{1,5, *}$, Suchankova $\mathrm{M}^{2, *}$, Ahrendt $\mathrm{K}^{1}$, Varga $\mathrm{L}^{1}$, Frajkova $\mathrm{Z}^{1}$, Urban $\mathrm{J}^{3}$, Kluckova $\mathrm{K}^{2}$, \\ Tedlova $\mathrm{E}^{4}$, Profant $\mathrm{M}^{1}$, Bucova $\mathrm{M}^{2}$
}

Department of Otorhinolaryngology and Head and Neck Surgery, Faculty of Medicine Comenius University and the University Hospital, Bratislava, Slovakia. miro.tedla@gmail.com

\begin{abstract}
PURPOSE: Chronic sinusitis can result from variable types of immune-mediated process, whose pathogenesis is not fully understood. Triggering receptors expressed on myeloid cells 1 and 2 (TREM-1, TREM-2) are involved in myeloid cell activation enabling these cells to fine-tune the inflammatory response, which may have an impact on subsequent adaptive immunity and may be the key factor in pathogenesis. The aim of the study was to analyse soluble TREM-1 and TREM-2 molecules in maxillary sinus lavage fluid and compare the defined subgroups selected from patients with chronic sinusitis with/without nasal polyps and allergy (asthma and allergic rhinitis).

METHODS: The levels of soluble TREM-1 and TREM-2 were measured by Elisa test in a cohort of patients with chronic maxillary sinusitis $(n=45)$. We compared subgroups of patients with nasal polyps $(n=33)$ and allergy $(n=25$ : inclusive of asthma $(n=11)$ and allergic rhinitis $(n=14))$ with the control group of patients without nasal polyps $(n=13)$, and without allergy $(n=21)$.

RESULTS: The study did not prove the difference between subgroups with and without nasal polyps. The levels of soluble TREM-1 did not differ significantly between patients with allergy (asthma and allergic rhinitis) and the control group without allergy $(p=0.4804)$. The levels of soluble TREM-2 were significantly higher in patients with allergy $(p=0.0028)$, asthma $(p=0.0103)$ and allergic rhinitis $(p=0.0137)$ as compared with the control group.

CONCLUSION: Our results suggest the role of TREM-2-mediated activation of myeloid cells in chronic sinusitis accompanied by allergy, asthma, and allergic rhinitis (Tab. 6, Ref. 25). Text in PDF www.elis.sk KEY WORDS: TREM-1 and TREM- 2 receptors, allergy, hypersensitive reaction, maxillary sinus lavage, bronchial asthma.
\end{abstract}

\section{Introduction}

Chronic maxillary sinusitis is a persisting inflammatory process of heterogeneous origin. Depending on aetiology, inflammation may be accompanied by various types of innate and adaptive immune responses leading to similar clinical presentations. Although two main forms, one with and one without polyps, were described $(1,2)$, this classification does not consider the underlying patho-

${ }^{1}$ Department of Otorhinolaryngology and Head and Neck Surgery, Faculty of Medicine Comenius University and the University Hospital, Bratislava, Slovakia, ${ }^{2}$ Institute of Immunology, Faculty of Medicine Comenius University Bratislava, Slovakia, ${ }^{3}$ National Institute for Tuberculosis Lung Diseases and Thoracic Surgery, Vysne Hagy, Slovakia, ${ }^{4}$ Department of Pneumology and Phtiseology, Comenius University Faculty of Medicine and University Hospital, Bratislava, Slovakia, and ${ }^{5}$ Institute of Cancer and Genomic Sciences, University of Birmingham, Birmingham, United Kingdom

Address for correspondence: M. Tedla, MD, MPH, PhD, Department of Otorhinolaryngology and Head and Neck Surgery, Faculty of Medicine Comenius University and the University Hospital, Bratislava, Antolska 11, SK-851 07 Bratislava, Slovakia. Phone: +421.905303007

Acknowledgement: This work was supported by the grant VEGA 1/0212/17.

* The author should be considered joint first author. genesis. Cellular and molecular mechanisms may vary between patients, and this may contribute to the difficulty of establishing a repeating and verifiable classification. The heterogeneity of pathogenesis can influence the clinical phenotype and response to treatment.

The sinonasal epithelium, mucociliary clearance, antimicrobial compound secretion per innate immune cells must react to large amounts of inhaled pathogens, allergens, and other airborne irritants. The appropriate reaction of the immune system is required for elimination of pathogens and restoration of homeostasis to be effective. On the other hand, inadequate reaction to commensals or allergens can lead to a failure in mechanisms of tolerance, hypersensitivity or hyperinflammation. The abnormal immune response can lead to recurrent and/or persistent inflammation of the paranasal sinuses.

Numerous studies have described microbes as an etiological factor (3-8) without a single causative agent associated with the disease. Some studies show an increased abundance and decreased diversity of the nasal microbiome $(9,10)$. In a large group of patients, sinusitis is associated with allergy. The presence of polyps does not reflect the type of inflammation; the frequency of polyps in allergic patients was not higher than in nonallergic ones (11, 
Tab. 1. Study groups.

\begin{tabular}{|c|c|c|c|c|c|c|c|}
\hline Diagnosis & $\mathrm{n}$ & Diagnosis (subgroup) & $\mathrm{n}$ & Diagnosis (subgroup) & $\mathrm{n}$ & Diagnosis (subgroup) & $\mathrm{n}$ \\
\hline \multirow{3}{*}{$\begin{array}{l}\text { Chronic maxillary } \\
\text { sinusitis }\end{array}$} & \multirow{3}{*}{46} & \multirow{2}{*}{$\begin{array}{l}\text { Chronic allergic } \\
\text { maxillary sinusitis }\end{array}$} & \multirow{2}{*}{25} & $\begin{array}{l}\text { Allergic rhinitis in non- } \mathrm{AB} \\
\text { patients }\end{array}$ & 14 & $\begin{array}{l}\text { with polyps } \\
\text { without polyps }\end{array}$ & $\begin{array}{r}10 \\
4\end{array}$ \\
\hline & & & & $\begin{array}{l}\text { Allergic rhinitis in } \mathrm{AB} \\
\text { patients }\end{array}$ & 11 & $\begin{array}{l}\text { with polyps } \\
\text { without polyps }\end{array}$ & $\begin{array}{l}9 \\
2\end{array}$ \\
\hline & & $\begin{array}{l}\text { Chronic maxillary sinusitis } \\
\text { without allergy }\end{array}$ & 21 & & & $\begin{array}{l}\text { with polyps } \\
\text { without polyps }\end{array}$ & $\begin{array}{r}14 \\
7\end{array}$ \\
\hline
\end{tabular}

$\mathrm{AB}$ - bronchial asthma

12); eosinophilia can be found in patients with or without polyps, respectively (13).

The allergic form of sinusitis is driven by the Th2 cells and type 2 innate lymphoid cells. Type 2 cytokines activate mast cells, basophils, and eosinophils, which leads to type I hypersensitivity reaction. To date, it is not fully explained how immunopathogenic mechanisms lead to the breakdown of tolerogenic pathways and shift the physiological response. The key factor could be found in the innate immunity response, which precedes the development of adaptive immunity and determines its intensity and direction.

Triggering receptors expressed on myeloid cells 1 and 2 (TREM-1, TREM-2) are recently discovered receptors, and their function is to fine-tune the activation of cells. TREM-1 intensifies the proinflammatory response by activating the intracellular pathways that lead to the increase in the production of proinflammatory cytokines and expression of costimulatory molecules. TREM-1 receptor's expression rapidly increases in the presence of inflammation caused by extracellular pathogenic bacteria or fungi $(14,15)$, and/or inflammation accompanied by necrosis, and the signals released from necrotic cells might serve as a potential ligand for TREM-1 (16). TREM-2 regulates the activation of dendritic cells (DC), macrophages, osteoclasts, and microglial cells. TREM-2/DAP12-mediated signalling promotes partial DC maturation and upregulation of cell surface expression of T-cell stimulatory molecules such as major histocompatibility complex (MHC) class II, CD40, and CD86 and CC chemokine receptor 7, which is crucial for DC migration to the lymph nodes (17). Contrary to TREM-1, it is TREM-2 that plays a role in inhibiting TNF and IL-6 production by macrophages and is responsible for DAP12-mediated inhibition of inflammatory responses induced by TLR ligands in macrophages. Strikingly, the TREM-2/DAP12 pathway may provide both inhibitory and activating signals depending on their microenvironment. Moreover, the relationship between TREM-2, DAP12, and biological functions may depend on the influence of additional DAP12-associated receptors and on the presence of TREM-2 ligands with a variable avidity/affinity, which may induce either activating or inhibitory signals through TREM-2/DAP12 (18). Helming et al demonstrated a DAP12-facilitated macrophage fusion leading to the formation of multinucleated giant cells (19).

Given the role of these receptors in orchestrating the immune response, a better understanding of their role in various types of inflammation appears to be essential in advancing our knowledge of underlying mechanisms of airway inflammation. Little is known about TREM expression in the airways, and it is still unclear if the receptor may play a role in the pathogenesis of allergy. In this study, we examined the levels of soluble forms of TREM-1 and TREM-2 molecules in the maxillary lavage fluid of patients with various forms of chronic maxillary sinusitis.

\section{Patients and methods}

\section{Study population}

Forty-six patients with chronic maxillary sinusitis were prospectively enrolled in the study. The study group contained 25 patients with allergy, out of whom 14 patients were with allergic rhinitis and 11 patients with bronchial asthma (AB), 33 patients with nasal polyps, and 13 patients without polyps. The control group consisted of 21 patients without any form of allergy (Tab. 1). The allergy status was confirmed by clinical and laboratory diagnostic methods provided by the managing Consultant Immunoallergologist.

The study was approved by the local Ethics Committee of the Faculty of Medicine, Comenius University in Bratislava, and written informed consent was obtained from all patients.

\section{Maxillary sinus lavage fluid processing}

The lavage procedure was carried out at the Department of Otorhinolaryngology and Head and Neck Surgery, Faculty of Medicine, Comenius University Bratislava, Slovakia during elective functional endoscopic sinus surgery. Maxillary antrum washout was performed as the first step after careful opening of the maxillary sinus to avoid bleeding. A volume of $5 \mathrm{ml}$ of sterile normal saline was instilled into the maxillary sinus and aspirated thereafter by gentle suction using a sterile syringe. The harvested maxillary sinus lavage fluid was immediately transported to the laboratory of the Institute of Immunology, Faculty of Medicine Comenius University Bratislava for analysis. The maxillary lavage fluid was first filtered through a double layer of sterile gauze, then centrifuged at $1,000 \mathrm{~g}$ for $20 \mathrm{~min}$ at $4{ }^{\circ} \mathrm{C}$, and the supernatants were collected and stored at $-80^{\circ} \mathrm{C}$ until analysis. The levels of sTREM-1 and sTREM-2 were measured with a sandwich enzyme-linked immunosorbent assay (human s TREM-1 and human sTREM-2 ELISA; Wuhan Fine Biotech Co., Ltd) according to the instructions of the manufacturer. 
Tab. 2. The levels of sTREM-1 in chronic maxillary sinusitis in patients with and without allergy.

\begin{tabular}{lll}
\hline & $\begin{array}{l}\text { sTREM-1 pg/ml } \\
\text { (in maxillary lavage fluid) }\end{array}$ & p* \\
\hline $\begin{array}{l}\text { Chronic maxillary } \\
\text { sinusitis with allergy } \\
(\mathrm{n}=25)\end{array}$ & $\begin{array}{l}\text { Mean } \pm \text { SD: } 174.9 \pm 220.8 \\
\text { Median: } 112.9\end{array}$ & \\
\hline $\begin{array}{l}\text { Chronic maxillary } \\
\text { sinusitis without allergy } \\
(\mathrm{n}=21)\end{array}$ & $\begin{array}{l}\text { Meange: } 0.97-956.7 \\
\text { Median: } 79.3\end{array}$ & \\
*Unpaired Student's t-test & Range: $12.2-8904.8$ & \\
\end{tabular}

Tab. 3. The levels of sTREM-2 in chronic maxillary sinusitis with and without allergy.

\begin{tabular}{lll}
\hline & $\begin{array}{l}\text { sTREM-2 pg/ml } \\
\text { (in maxillary lavage fluid) }\end{array}$ & $\mathrm{p}^{*}$ \\
\hline $\begin{array}{l}\text { Chronic maxillary } \\
\text { sinusitis with allergy } \\
(\mathrm{n}=25)\end{array}$ & $\begin{array}{l}\text { Mean } \pm \text { SD: } 5757.3 \pm 2340.0 \\
\text { Median: } 5817.6\end{array}$ & \\
\hline $\begin{array}{l}\text { Chronic maxillary } \\
\text { sinusitis without allergy } \\
(\mathrm{n}=21)\end{array}$ & $\begin{array}{l}\text { Range: } 774.07-9566.1 \\
\text { Mean } \pm \text { SD: } 3519.5 \pm 2438.8\end{array}$ & \\
\hline
\end{tabular}

*Unpaired Student's T-test

\section{Statistical analysis}

The one-sample Kolmogorov-Smirnov test was used to determine whether the investigated populations followed normal distribution. Either non-parametric Mann-Whitney U- test or parametric non-paired t-test with Welch correction was used to determine the difference and the statistical significance.

\section{Results}

The levels of maxillary lavage fluid's sTREM-1 did not differ significantly between the patients with allergy and the control group

We compared sTREM-1 concentrations in maxillary lavage fluid in patients with chronic maxillary sinusitis accompanied by allergy with the control group of patients with chronic maxillary sinusitis without confirmed allergy. The patients with allergy had slightly higher concentrations of STREM-1 as compared to the control group, however, the difference was not statistically significant ( $\mathrm{p}=0.4804)$ (Tab. 2).

The levels of maxillary lavage fluid's STREM-2 were increased in patients with allergy compared with the control group of nonallergic patients

The concentrations of STREM-2 in maxillary lavage fluid in allergic patients with chronic maxillary sinusitis were significantly higher than the levels of sTREM-2 in the control group of patients with chronic maxillary sinusitis without confirmed allergy $(p=0.0028)$ (Tab. 3).

Furthermore, we selected two subgroups of patients with $\mathrm{AB}$ and allergic rhinitis from the group of patients with

*Unpaired Student's t-test
Tab. 4. The levels of maxillary lavage fluid STREM-2 in chronic maxillary sinusitis in patients with and without bronchial asthma.

\begin{tabular}{|c|c|c|}
\hline & $\begin{array}{l}\text { sTREM-2 pg/ml } \\
\text { (maxillary lavage fluid) }\end{array}$ & $\mathrm{p}^{*}$ \\
\hline Chronic maxillary & Mean \pm SD: $5973.5 \pm 2343.8$ & \multirow{6}{*}{0.0103} \\
\hline sinusitis in $\mathrm{AB}$ patients & Median: 6549.0 & \\
\hline$(\mathrm{n}=11)$ & Range: $1315.0-8849.0$ & \\
\hline Chronic maxillary & Mean \pm SD: $3519.5 \pm 2438.8$ & \\
\hline sinusitis without allergy & Median: 3196.8 & \\
\hline (control group) $(n=21)$ & Range: 0-7044.3 & \\
\hline
\end{tabular}

*Unpaired Student's t -test, AB - bronchial asthma

Tab. 5. The levels of sTREM-2 in maxillary lavage fluid in patients with chronic maxillary sinusitis with and without allergic rhinitis.

\begin{tabular}{lll}
\hline & sTREM-2 pg/ml & $\mathrm{p}^{*}$ \\
\hline Chronic maxillary & Mean \pm SD: $5548.8 \pm 1949.4$ & \\
sinusitis with allergic & Median: 5711.0 & \\
rhinitis ( $\mathrm{n}=14)$ & Range: $774.0-7859.5$ & \\
\hline Chronic maxillary & Mean \pm SD: $3519.5 \pm 2438.8$ & \\
sinusitis without allergy & Median: 3196.8 & \\
(control group) $(\mathrm{n}=21)$ & Range: $0-7044.3$ & \\
\hline *Unpaired Student's t-test & &
\end{tabular}

allergies. Both subgroups were then compared with the control group separately.

Thelevelsofmaxillarylavagefluid'ssTREM-2 were increased in $A B$ patients compared with the control group of non-allergic patients

The levels of sTREM-2 in maxillary lavage in bronchial asthma patients suffering from chronic maxillary sinusitis were significantly higher than in the control group of patients without allergy $(\mathrm{p}=0.0103)$ (Tab. 4).

The levels of maxillary lavage fluid's STREM-2 were increased in allergic rhinitis compared with the control group of patients without allergy

The tested levels of sTREM-2 in maxillary lavage fluid in patients with chronic maxillary sinusitis accompanied by allergic rhinitis were also significantly higher than sTREM-2 levels in the control group of patients without allergy $(p=0.0137)$ (Tab. 5).

The concentrations of sTREM-1 and sTREM-2 are not influenced by the presence of nasal polyps

Finally, we subdivided all patients of the group with chronic maxillary sinusitis into two subgroups, namely those with and

Tab. 6. The levels of sTREM-1 and sTREM-2 in maxillary lavage fluid in chronic maxillary sinusitis with and without nasal polyps.

\begin{tabular}{|c|c|c|c|c|}
\hline & sTREM-1 pg/ml & $\mathrm{p}^{*}$ & sTREM-2 pg/ml & $\mathrm{p}^{*}$ \\
\hline $\begin{array}{l}\text { Chronic maxillary } \\
\text { sinusitis with polyps } \\
(\mathrm{n}=33)\end{array}$ & $\begin{array}{l}\text { Mean } \pm \text { SD: } 144.4 \pm 182.8 \\
\text { Median: } 83.8 \\
\text { Range: } 1.0-891.8\end{array}$ & 2156 & $\begin{array}{l}\text { Mean } \pm \text { SD: } 4720.1 \pm 2584.9 \\
\text { Median: } 5116.7 \\
\text { Range: } 301.7-9566.1\end{array}$ & 94 \\
\hline $\begin{array}{l}\text { Chronic maxillary } \\
\text { sinusitis without polyps } \\
(\mathrm{n}=13)\end{array}$ & $\begin{array}{l}\text { Mean } \pm \text { SD: } 249.2 \pm 286.4 \\
\text { Median: } 168.8 \\
\text { Range: } 12.2-956.7\end{array}$ & 0.2150 & $\begin{array}{l}\text { Mean } \pm \text { SD: } 4791.7 \pm 2860.3 \\
\text { Median: } 6126.2 \\
\text { Range: } 0-7630.9\end{array}$ & 940 \\
\hline
\end{tabular}


without nasal polyps. Interestingly, we did not find any differences between these two subgroups of patients in the levels of either sTREM-1 or sTREM-2 in their maxillary lavage fluid (Tab. 6).

\section{Discussion}

Molecular mechanisms of innate immunity which are critical for correct tuning of the adaptive immune response are not clearly explained and may be the key factor participating in the pathogenesis of chronic sinusitis. Dendritic cells (DC), macrophages, or epithelial cells may express numerous types of molecules on their surfaces depending on various microenvironments. Membranes of these cells are equipped with pattern recognition receptors (PRR), cytokine receptors, immunoglobulin receptors, and other types of receptors. Activation through these receptors may initiate DC maturation, which results in migration to the regional lymph nodes, where they encounter and activate naive T cells. Depending on the microenvironment, DC mature and the cell surface expression of a series of molecules is up-regulated. These molecules might be crucial for subsequent activation and direction of the ensuing immune response. The expression of TREM receptors can modulate inflammatory responses by up-regulating the cell surface expression of T-cell stimulatory molecules (17) by influencing the PRRs activation. These could be the crucial mechanisms of antibacterial airway innate immunity and/or allergic airway inflammation that would result in priming and engaging the adaptive immune system (20-22).

The involvement of TREM receptors in the tuning of innate cell activation and subsequent abnormal adaptive immune response in allergy is not known.

To date, only a few studies suggested the role of TREM receptors in allergies. Hall et al (2017) found TREM-2 expression in the airways and on DC subsets isolated from the lungs and mediastinal lymph nodes in a murine model of allergic airway inflammation, and speculated that the induction of TREM-2 on DC subsets could indicate an impaired defence mechanism originally aimed at decreasing rather than enhancing the inflammation (8). Wang et al (2016) found that overexpression of TREM-2 in mice with allergic asthma significantly alleviated airway inflammation and airway remodelling (9). The study by Habibzay et al found fatal pneumococcal pneumonia due to impaired neutrophil recruitment as a result of airway exposure to house-dust mite in a sensitized mouse model of allergic airway disease. This was caused by desensitization of TLRs, up-regulation of endogenous negative regulators of TLRs (A20, IRAK) as well as preference of TREM-2 expression to inhibit TLR signalling (21). Based on these results, it could be speculated that the attenuation of the immune response due to TREM-2 may lead to colonization and survival of pathogenic microorganisms, microbiome disruption, and persistence of chronic inflammation. We have not found any studies focused on the role of TREM-1 in allergy. Only a few studies suggested its role in bronchial asthma $(23,24)$.

\section{Synopsis of new findings}

In this study, we analyzed the levels of sTREM molecules in maxillary lavage fluid in patients with chronic sinusitis and com- pared defined subgroups of patients with/without nasal polyps and allergy ( $\mathrm{AB}$ and allergic rhinitis). No statistically significant differences were found for the levels of sTREM-1. However, this molecule may also play a role in the immunopathogenesis of chronic sinusitis, while its role is probably not specific to allergic inflammation or nasal polyps. More importantly, we found significantly increased levels of sTREM-2 in patients with allergy, bronchial asthma, and allergic rhinitis as compared with the control group of non-allergic patients. Our results suggest that TREM-2 may play an important role in the pathogenesis of allergic inflammation as one of the pathogenetic mechanisms of chronic sinusitis in humans. This is in agreement with a recent study of Hall et al (2017) who point to this role in an animal models in mice (25). However, based on our study, the source of these soluble TREM molecules remains unclear. Similarly, we cannot explain if their role is causative or protective. Further studies are needed to clarify their role in allergic inflammation.

\section{Conclusion}

The obtained results suggest that allergies are associated with an increase in TREM-2 expression in patients with chronic allergic sinusitis. This is the first study describing the role of TREM-2 molecules in patients with allergies in humans. The question as to whether the TREM-2 expression in allergies is a causative factor or just a protective one requires further studies.

\section{References}

1. Meltzer EO, Hamilos DL, Hadley JA et al. Rhinosinusitis: Establishing definitions for clinical research and patient care. Otolaryngol Head Neck Surg 2004; 131: 1-62.

2. Fokkens WJ, Lund VJ, Hopkins C et al. European Position Paper on Rhinosinusitis and Nasal Polyps 2020. Rhinology 2020; 58 (Supplement 29): 1-464.

3. Bachert C, Gevaert P, Cauwenberge PV. Staphylococcus aureus enterotoxins: a key in airway disease? Allergy 2002; 57: 480-487.

4. Fokkens WJ, van Drunen C, Georgalas C, Ebbens F. Role of fungi in pathogenesis of chronic rhinosinusitis: the hypothesis rejected. Curr Opin Otolaryngol Head Neck Surg 2012; 20 (1): 19-23.

5. Ponikau JU, Sherris DA, Kephart GM, Adolphson C, Kita H. The role of ubiquitous airborne fungi in chronic rhinosinusitis. Curr Allergy Asthma Rep 2005; 5 (6): 472-476.

6. Schleimer RP. Immunopathogenesis of Chronic Rhinosinusitis and Nasal Polyposis. Annu Rev Pathol 2017; 12: 331-357.

7. Ramadan HH, Sanclement JA, Thomas JG. Chronic rhinosinusitis and biofilms. Otolaryngol Head Neck Surg 2005; 132: 414-417.

8. Sanderson AR, Leid JG, Hunsaker D. Bacterial biofilms on the sinus mucosa of human subjects with chronic rhinosinusitis. Laryngoscope 2006; 116 (7): 1121-1126.

9. Mahdavinia M, Keshavarzian A, Tobin MC, Landay AL, Schleimer RP. A comprehensive review of the nasal microbiome in chronic rhinosinusitis (CRS). Clin Exp Allergy 2016; 46 (1): 21-41. 
10. Chalermwatanachai T, Velásquez LC, Bachert C. The microbiome of the upper airways: focus on chronic rhinosinusitis. World Allergy Organ J 2015; 8 (1): 3.

11. Slavin RG. Allergy Is Not a Significant Cause of Nasal Polyps. Arch Otolaryngol Head Neck Surg 1992; 118 (7): 771.

12. Settipane GA, Chafee FH. Nasal polyps in asthma and rhinitis. A review of 6,037 patients. J Allergy Clin Immunol 1977; 59 (1): 17-21.

13. Kountakis SE, Arango P, Bradley D, Wade ZK, Borish L. Molecular and cellular staging for the severity of chronic rhinosinusitis. Laryngoscope 2004; 114 (11): 1895-1905.

14. Bouchon A, Dietrich J, Colonna M. Cutting edge: inflammatory responses can be triggered by TREM-1, a novel receptor expressed on neutrophils and monocytes. Journal of Immunology (Baltimore, Md: 1950) 2000; 164 (10): 4991-4995.

15. Bleharski JR, Kiessler V, Buonsanti $C$ et al. A role for triggering receptor expressed on myeloid cells-1 in host defense during the earlyinduced and adaptive phases of the immune response. Journal of Immunology (Baltimore, Md: 1950) 2003; 170 (7): 3812-3818.

16. El Mezayen R, El Gazzar M, Seeds MC, McCall CE, Dreskin SC, Nicolls MR. Endogenous signals released from necrotic cells augment inflammatory responses to bacterial endotoxin. Immunol Lett 2007; 111 (1): 36-44.

17. Bouchon A, Hernández-Munain C, Cella M, Colonna M. A DAP12mediated pathway regulates expression of $\mathrm{CC}$ chemokine receptor 7 and maturation of human dendritic cells. The Journal of Experimental Medicine 2001; 194 (8): 1111-1122.
18. Paradowska-Gorycka A, Jurkowska M. Structure, expression pattern and biological activity of molecular complex TREM-2/DAP12. Human Immunology 2013; 74 (6): 730-737.

19. Helming L, Tomasello E, Kyriakides TR et al. Essential role of DAP12 signaling in macrophage programming into a fusion-competent state. Science Signaling 2008; 1 (43): ra11.

20. Hariri BM, Cohen NA. New insights into upper airway innate immunity. Am J Rhinol Allergy 2016; 30 (5): 319-323.

21. Habibzay M, Saldana JI, Goulding J, Lloyd CM, Hussell T. Altered regulation of Toll-like receptor responses impairs antibacterial immunity in the allergic lung. Mucosal Immunol 2012; 5 (5): 524-534.

22. Hall SC, Agrawal DK. Toll-like receptors, triggering receptor expressed on myeloid cells family members and receptor for advanced glycation end-products in allergic airway inflammation. Expert Rev Respir Med 2016; 10 (2): 171-184.

23. Bucova M, Suchankova M, Dzurilla $M$ et al. Inflammatory marker sTREM-1 reflects the clinical stage and respiratory tract obstruction in allergic asthma bronchiale patients and correlates with number of neutrophils. Mediators of Inflammation 2012: 628754.

24. Buckland KF, Ramaprakash H, Murray LA et al. Triggering receptor expressed on myeloid cells-1 (TREM-1) modulates immune responses to Aspergillus fumigatus during fungal asthma in mice. Immunological Investigations 2011; 40 (7-8): 692-722.

25. Hall SC, Agrawal DK. Increased TREM-2 expression on the subsets of $\mathrm{CD} 11 \mathrm{c}+$ cells in the lungs and lymph nodes during allergic airway inflammation. Scientific Reports 2017; 7 (1): 11853.

Received October 21, 2020. Accepted November 25, 2020. 